\title{
DPPH Scavenging Assay of Eighty Four Bangladeshi Medicinal Plants
}

\author{
Nizam Uddin ${ }^{1}$, Rakibul Islam ${ }^{1}$, Nahid Hasan ${ }^{2}$, Md. Sazzad Hossain ${ }^{1}$, Aumit \\ Roy $^{2}$, Md. Monir Hossain ${ }^{2}$, Md. Sohel Rana ${ }^{2}$ \\ ${ }_{1,2}$ Laboratory of Natural Products Research, Jahangirnagar University, Savar, Dhaka-1342, Bangladesh
}

\begin{abstract}
This study was designed to screen out free radical scavenging potentiality of 84 medicinal plants. Stock solution of different plant extracts and standard were diluted to achieve suitable concentrations. A control was also prepared without plant extract solution. Then 0.004\% DPPH solution was added. The mixtures were incubated in the room temperature for 30 minutes. Then the absorbance was measured at $517 \mathrm{~nm}$ against solvent in UV-spectrophotometer and then IC50 was calculated. In this experiment two standard were usedascorbic acid and BHT. Both showed a significant IC50 value of $15.5 \mu \mathrm{g} / \mathrm{mL}$, and $46.54 \mu \mathrm{g} / \mathrm{mL}$ respectively. Among 84 medicinal plants Syzygim cumini, Casuarina littorea, Borassus flabellifer, Enhydra fluctuans, and Minusops elengi exhibited highest radical scavenging potential with an IC50 value of $12.816 \mu \mathrm{g} / \mathrm{mL}$, $14.467 \mu \mathrm{g} / \mathrm{mL}, 15.755 \mu \mathrm{g} / \mathrm{mL}, 15.653 \mu \mathrm{g} / \mathrm{mL}$, and $20.380 \mu \mathrm{g} / \mathrm{mL}$ respectively. All these value are very close to the IC50 value of ascorbic acid and better than IC50 value of BHT (Butylated Hydroxy Toluene). Syzygim cumini is the most powerful scavenger among all tested medicinal plants and also most strong scavenger than ascorbic acid and BHT. Scavenging activity was found to increase in dose dependent manner. Another 30 medicinal plants exhibited good scavenging property and 14 medicinal plants showed moderate scavenging activity. The rest presented lower scavenging activity. This present study indicates that plants having good scavenging property may have various health beneficial effects and these plants can be considered as valuable source of bioactive components with high antioxidant properties.
\end{abstract}

Keywords: Antioxidant, Ascorbic acid, BHT, DPPH, Medicinal plants

\section{Introduction}

Oxygen, no doubt, the $3^{\text {rd }}$ most elements in the universe after Hydrogen and Helium and the most abundant element by mass in earth crust. It plays a vital role in our breathing process. Living organism requires ample amount of oxygen for energy production and body metabolism process. All metabolic processes in our body depend on oxygen[1]. This indispensable element sometimes causes serious oxygen toxicity by formation of reactive oxygen species (ROS) which represents both free and non free radicals. Some common types of encountered free radicals are hydroxyl radical $(\mathrm{HO} \bullet)$, superoxide radical $\left(\mathrm{O}_{2} \bullet-\right)$, nitric oxide radical $(\mathrm{NO} \bullet)$, and lipid peroxyl radical (LOO•) while non-free radical species principally being $\mathrm{H}_{2} \mathrm{O}_{2}$, singled oxygen $\left(1 \mathrm{O}_{2}\right)$, and hypoclorus acid $(\mathrm{HOCl})[2]$. Free radical damage is closely related to oxidative damage. These types of species are involved in activation of many types of procarcinogens and promutagens. Free radicals react with biomolecules like DNA, RNA, lipid, and protein and result in gene mutation and cell structure damage and lead to development of cancer, cardiovascular disorder, neurodegenerative disease (Alzheimer's disease, Parkinson's disease), atherosclerosis, liver injury, diabetes mellitus[3,4]. Reactive oxygen species (ROS) exerts oxidative stress towards the cells of human to face 10000 oxidative hit per second and when this type of hit breaks antioxidant defense mechanism, the free radicals then attack cell macromolecules and lead to a number of physiological disorders[4].

Bangladesh has rich plant and animal sources in particular reference to the antioxidant compounds from medicinal plants. So, well planned, careful, innovative, factual, and systemic research will be of great benefit to our poor people who are the helpless victims to various physiological diseases caused by free radicals. This study performed on medicinal plants support the proposal that plant constituents with antioxidant properties show good free radical and non free radical scavenging property in our biological system[5]. During our experiment we studied eighty four medicinal plants for evaluating DPPH (1, 1-diphenyl-2 picryl hydrazyl) scavenging assay. The assessment is interesting and useful task for finding out potential natural antioxidants.

\subsection{Chemicals and drugs:}

\section{Materials and Methods}

DPPH (1, 1-diphenyl-2 picryl hydrazyl) was obtained from Sigma chemical Co.USA. Ascorbic acid was obtained from SD Fine chem. Ltd. Biosar, India. 


\subsection{Preparation of plant materials:}

All the plants were collected from different districts and villages of Bangladesh. Most of the samples were identified by experts in Bangladesh National Herbarium, Mirpur, Dhaka and the rest by Professor Dr. Abdul Ghani (Professor of Pharmacognosy, Stamford University Bangladesh)[6]. The specimen samples are kept in the Bangladesh National Herbarium. Suitable extraction procedures were used for these plants in different solvents.

\subsection{DPPH free radical scavenging assay:}

The free radical scavenging activity of the extracts, based on the scavenging activity of the stable 1, 1diphenyl-2 picryl hydrazyl (DPPH) free radical was determined by the method described[7]. Solution of plant extracts of various concentrations were properly mixed with $0.004 \%$ methanol solution of DPPH. Absorbance at $517 \mathrm{~nm}$ was determined after $30 \mathrm{~min}$, and the percentage inhibition activity was calculated from the equation $\left[\left(\mathrm{A}_{0}-\mathrm{A}_{1}\right) / \mathrm{A}_{0}\right] \times 100$ i.e., (1)

Where, $\mathrm{A}_{0}$ is the absorbance of the control, and $\mathrm{A}_{1}$ is the absorbance of the extract/ standard.

\subsection{Statistical analysis:}

The inhibition curves were prepared and IC50 values were obtained with the help of Microsoft Excel 2007.

\section{Result}

Unlike other free radicals such as hydroxide ion or superoxide anion DPPH has an advantage of being unaffected by certain side reaction such as enzyme inhibition and metal ion chelation process[7]. DPPH solution exhibits a deep purple color with absorption maximum at $517 \mathrm{~nm}$. Generally, the color disappears when antioxidant compounds present in the medium. For this research prospect, 84 different types of plants were tested and screened for DPPH scavenging assay. Results of all plants are given below in a table. For each plant, eight types of information were provided including scientific name, family, local name, english name, traditional use, part used for assay, name of solvent used and $\mathrm{IC}_{50}$ value. Most of the morphological information were collected from Ghani A 2003[6].

TABLE 1: $\mathrm{IC}_{50}$ Value of 84 Medicinal Plants with their Traditional uses and Botanical Identity

\begin{tabular}{|c|c|c|c|c|c|c|c|c|}
\hline $\begin{array}{l}\text { Sl } \\
\text { no }\end{array}$ & Scientific name & Family & $\begin{array}{l}\text { Local } \\
\text { name }\end{array}$ & $\begin{array}{l}\text { English } \\
\text { name }\end{array}$ & Traditional use & $\begin{array}{l}\text { Part } \\
\text { used }\end{array}$ & Solvent & $\begin{array}{l}\mathrm{IC}_{50} \\
\text { value } \\
(\mu \mathrm{g} / \mathrm{mL})\end{array}$ \\
\hline \multirow{4}{*}{01} & \multirow{4}{*}{$\begin{array}{l}\text { Pistia stratiotes } \\
\text { Linn. }\end{array}$} & \multirow{4}{*}{ Araceae } & \multirow{4}{*}{$\begin{array}{l}\text { Topa } \\
\text { pana, } \\
\text { Toka pana }\end{array}$} & \multirow{4}{*}{$\begin{array}{l}\text { Water } \\
\text { cabbage }\end{array}$} & \multirow{4}{*}{$\begin{array}{l}\text { Used } \\
\text { tuberculosis, ear } \\
\text { disease }\end{array}$} & \multirow{2}{*}{ Root } & Methanol & 3103.75 \\
\hline & & & & & & & Pet ether & 12700 \\
\hline & & & & & & \multirow{2}{*}{ Leaf } & Methanol & 963.846 \\
\hline & & & & & & & Pet ether & 4698.33 \\
\hline 02 & $\begin{array}{l}\text { Mikania } \\
\text { scandens } \\
\text { (L).willd }\end{array}$ & Asteraceae & Asamlata & Heartleaf & $\begin{array}{l}\text { Used to treat } \\
\text { stomach ulcer, } \\
\text { inflammation }\end{array}$ & Leaf & $\begin{array}{l}\text { Hydro } \\
\text { methanol }\end{array}$ & 104.1010 \\
\hline 03 & $\begin{array}{l}\text { Punica } \\
\text { granatum }\end{array}$ & Punicaceae & $\begin{array}{l}\text { Dalim, } \\
\text { Anar }\end{array}$ & $\begin{array}{l}\text { Pomegran } \\
\text { ate }\end{array}$ & $\begin{array}{l}\text { Antidiarrhoel, } \\
\text { anthelmintic }\end{array}$ & Rind & $\begin{array}{l}\text { Hydro } \\
\text { methanol }\end{array}$ & 35.558 \\
\hline 04 & $\begin{array}{l}\text { Xanthium } \\
\text { induicm } \\
\text { koeing }\end{array}$ & Asteraceae & Ghagra & $\begin{array}{l}\text { Rough } \\
\text { cocklebur }\end{array}$ & $\begin{array}{l}\text { Used in urinary } \\
\text { compliant, sore of } \\
\text { lips and mouth }\end{array}$ & Leaf & $\begin{array}{l}\text { Hydro } \\
\text { methanol }\end{array}$ & 70.684 \\
\hline 05 & Tinospoa crispa & $\begin{array}{l}\text { Menisper- } \\
\text { maceae }\end{array}$ & $\begin{array}{l}\text { Gada- } \\
\text { ncha } \\
\text { gachh }\end{array}$ & $\begin{array}{l}\text { Heavenly } \\
\text { elexir }\end{array}$ & $\begin{array}{l}\text { Jaundice, } \\
\text { rheumatic fever, } \\
\text { ulcer }\end{array}$ & $\begin{array}{l}\text { Aerial } \\
\text { part }\end{array}$ & Methanol & 90.074 \\
\hline 06 & $\begin{array}{l}\text { Syzygim cumini } \\
\text { (Linn.) }\end{array}$ & Myrtaceae & $\begin{array}{l}\text { Deshijam, } \\
\text { Kalojam }\end{array}$ & $\begin{array}{l}\text { Black } \\
\text { plum }\end{array}$ & $\begin{array}{l}\text { Used in diarrhea, } \\
\text { diabetes: used as } \\
\text { gargle, mouthwash }\end{array}$ & Leaf & Methanol & 12.816 \\
\hline 07 & $\begin{array}{l}\text { Michelia } \\
\text { champaca }(\mathrm{L} .)\end{array}$ & $\begin{array}{l}\text { Magno- } \\
\text { liaceae }\end{array}$ & $\begin{array}{l}\text { Swarna } \\
\text { champa }\end{array}$ & Champaca & $\begin{array}{l}\text { Expectorant, } \\
\text { stimulant, anti } \\
\text { inpflammatory }\end{array}$ & Leaf & Methanol & 67.639 \\
\hline 08 & $\begin{array}{l}\text { Ipomoea } \\
\text { quamoclit (L.) }\end{array}$ & $\begin{array}{l}\text { Convol- } \\
\text { vulaceae }\end{array}$ & Kunjolata & Star glory & $\begin{array}{l}\text { Applied to } \\
\text { carbuncles, and } \\
\text { bleeding piles, } \\
\text { treatment of ulcer } \\
\text { and breast pain }\end{array}$ & $\begin{array}{l}\text { Aerial } \\
\text { Part }\end{array}$ & $\begin{array}{l}\text { Hydro- } \\
\text { Methanol }\end{array}$ & 78.284 \\
\hline 09 & $\begin{array}{l}\text { Hopea odorata } \\
\text { roxb }\end{array}$ & Dipterocarpaceae & Telsur & $\begin{array}{l}\text { White } \\
\text { thingan }\end{array}$ & $\begin{array}{l}\text { Used in gingivitis, } \\
\text { as an ointment for } \\
\text { sores and wounds }\end{array}$ & Leaf & $\begin{array}{l}\text { Hydro- } \\
\text { Methanol }\end{array}$ & 99.604 \\
\hline 10 & $\begin{array}{l}\text { Feronia lomolia } \\
\text { Linn. }\end{array}$ & Rutaceae & Kodbel & $\begin{array}{l}\text { Wood } \\
\text { apple }\end{array}$ & $\begin{array}{l}\text { Remedy for } \\
\text { venomous insect, } \\
\text { and reptiles }\end{array}$ & $\begin{array}{l}\text { Aerial } \\
\text { part }\end{array}$ & $\begin{array}{l}\text { Hydro- } \\
\text { Methanol }\end{array}$ & 53.073 \\
\hline
\end{tabular}


DPPH Scavenging Assay of Eighty Four Bangladeshi Medicinal Plants

\begin{tabular}{|c|c|c|c|c|c|c|c|c|}
\hline 11 & $\begin{array}{l}\text { Curcuma } \\
\text { alismatifolia } \\
\text { Gangnep. }\end{array}$ & Zingiberaceae & & $\begin{array}{l}\text { Summer } \\
\text { tulip }\end{array}$ & $\begin{array}{l}\text { Applied to bruise, } \\
\text { sprain, snake bite }\end{array}$ & Leaf & $\begin{array}{l}80 \% \\
\text { methanol }\end{array}$ & 58.4513 \\
\hline \multirow{4}{*}{12} & \multirow{4}{*}{$\begin{array}{l}\text { Commelina } \\
\text { benghalensis } \\
\text { Linn. }\end{array}$} & \multirow{4}{*}{ Commelinaceae } & \multirow{4}{*}{$\begin{array}{l}\text { Dholpata, } \\
\text { Kanchira }\end{array}$} & \multirow{4}{*}{$\begin{array}{l}\text { Venus } \\
\text { bath }\end{array}$} & \multirow{4}{*}{$\begin{array}{l}\text { Demulcent, } \\
\text { emollient, laxative }\end{array}$} & \multirow{4}{*}{$\begin{array}{l}\text { Aerial } \\
\text { part }\end{array}$} & Chloroform & 28.9087 \\
\hline & & & & & & & Pet ether & 43.7936 \\
\hline & & & & & & & n-butanol & 106.285 \\
\hline & & & & & & & $\begin{array}{l}\text { Hydrometh } \\
\text { anol }\end{array}$ & 78.7301 \\
\hline 13 & $\begin{array}{l}\text { Cocos nucifera } \\
\text { Linn. }\end{array}$ & Arecaceae & $\begin{array}{l}\text { Narikel, } \\
\text { Daab }\end{array}$ & Coconut & $\begin{array}{lr}\text { Used in } & \text { dysentery, } \\
\text { hair } & \text { nutrient } \\
\text { alopecia } & \end{array}$ & Kernel & $\begin{array}{l}80 \% \\
\text { methanol }\end{array}$ & 41.2227 \\
\hline 14 & $\begin{array}{l}\text { Caesalpinia } \\
\text { plucherrima } \\
\text { Linn. }\end{array}$ & Caesalpiniceae & $\begin{array}{l}\text { Krisnachu } \\
\text { ra }\end{array}$ & $\begin{array}{l}\text { Peacock } \\
\text { flower }\end{array}$ & $\begin{array}{l}\text { Liver disorder, } \\
\text { cough, bronchitis, } \\
\text { asthma }\end{array}$ & Leaf & $\begin{array}{l}\text { Hydrometh } \\
\text { anol }\end{array}$ & 48.2490 \\
\hline 15 & $\begin{array}{l}\text { Butea } \\
\text { monosperma } \\
\text { (Lam) Taub } \\
\end{array}$ & Papilionaceae & Polash & $\begin{array}{l}\text { Flame of } \\
\text { forest }\end{array}$ & $\begin{array}{l}\text { Used as anti } \\
\text { ovulatory, } \\
\text { anthelmintic, } \\
\text { laxative }\end{array}$ & Leaf & $\begin{array}{l}\text { Hydrometh } \\
\text { anol }\end{array}$ & 78.284 \\
\hline 16 & $\begin{array}{l}\text { Baccurea } \\
\text { ramiflora Lour. }\end{array}$ & Phyllanthaceae & Lotkon & $\begin{array}{l}\text { Burmese } \\
\text { grape }\end{array}$ & $\begin{array}{l}\text { Young leaves as } \\
\text { vegetables, } \\
\text { flavoring agent } \\
\text { with curries }\end{array}$ & $\begin{array}{l}\text { Fruit } \\
\text { pericarp }\end{array}$ & $\begin{array}{l}\text { Hydrometh } \\
\text { anol }\end{array}$ & 94.6248 \\
\hline \multirow{3}{*}{17} & \multirow{3}{*}{$\begin{array}{l}\text { Artocarpus } \\
\text { lacucha Buch. } \\
\text { Ham }\end{array}$} & \multirow{3}{*}{ Moraceae } & \multirow{3}{*}{ Daowa } & \multirow{3}{*}{$\begin{array}{l}\text { Monkey } \\
\text { jack }\end{array}$} & \multirow{3}{*}{$\begin{array}{l}\text { Applied to pimple, } \\
\text { cracked skin and } \\
\text { sores }\end{array}$} & Pulp & Methanol & 24.9315 \\
\hline & & & & & & Pericarp & Methanol & 33.501 \\
\hline & & & & & & Leaf & Methanol & 50.5641 \\
\hline 18 & $\begin{array}{l}\text { Cyperus } \\
\text { rotundus Linn. }\end{array}$ & Cyperaceae & $\begin{array}{l}\text { Motha, } \\
\text { Mustak }\end{array}$ & Nut grass & $\begin{array}{l}\text { Active against } \\
\text { kapha and pitta } \\
\text { suppressant, cure } \\
\text { infection }\end{array}$ & $\begin{array}{l}\text { Whole } \\
\text { bush }\end{array}$ & Ethanol & 35.9439 \\
\hline 19 & $\begin{array}{l}\text { Dillenia indica } \\
\text { Linn. }\end{array}$ & Dilleniaceae & Chalta & $\begin{array}{l}\text { Elephant } \\
\text { tree }\end{array}$ & $\begin{array}{lr}\text { Used in } & \text { stomach } \\
\text { disorder; } & \text { anti } \\
\text { microbial } & \end{array}$ & Leaf & Methanol & 32.622 \\
\hline 20 & Brassica nigra & Brassicaceae & $\begin{array}{l}\text { Kalo } \\
\text { sorisa }\end{array}$ & $\begin{array}{l}\text { Black } \\
\text { mustard }\end{array}$ & $\begin{array}{l}\text { Seedlings used in } \\
\text { salad; plant used in } \\
\text { rheumatism }\end{array}$ & $\begin{array}{l}\text { Whole } \\
\text { plant }\end{array}$ & Ethanol & 63.045 \\
\hline 21 & $\begin{array}{l}\text { Raphanus } \\
\text { sativus } \\
\text { var.sativus } \\
\end{array}$ & Brassicaceae & Mula & Raddish & $\begin{array}{l}\text { Added in salad; } \\
\text { have tonic and } \\
\text { laxative effect }\end{array}$ & $\begin{array}{l}\text { Celery } \\
\text { with root }\end{array}$ & Ethanol & 398.11 \\
\hline 22 & $\begin{array}{l}\text { Raphanus } \\
\text { sativus } \\
\text { subsp.longi } \\
\text { pinnatus } \\
\end{array}$ & Brassicaceae & Sada mula & $\begin{array}{l}\text { White } \\
\text { raddish }\end{array}$ & $\begin{array}{l}\text { White radish juice } \\
\text { used to expel } \\
\text { phlegm }\end{array}$ & $\begin{array}{l}\text { Celery } \\
\text { with root }\end{array}$ & Ethanol & 398.107 \\
\hline 23 & $\begin{array}{l}\text { Ficus racemosa } \\
\text { (L.) }\end{array}$ & Moraceae & Dumur & $\begin{array}{l}\text { Cluster } \\
\text { tree }\end{array}$ & $\begin{array}{l}\text { Latex alleviate } \\
\text { edema; applied in } \\
\text { skin }\end{array}$ & Fruit & Ethanol & 8459.663 \\
\hline \multirow{3}{*}{24} & \multirow{3}{*}{$\begin{array}{l}\text { Geodorum } \\
\text { densiflorum } \\
\text { (Lam) Schltr }\end{array}$} & \multirow{3}{*}{ Orchidaceae } & \multirow{3}{*}{$\begin{array}{l}\text { Shonkho } \\
\text { mul, } \\
\text { Kukumari } \\
\text { a }\end{array}$} & & \multirow{3}{*}{$\begin{array}{l}\text { Diarrhea, } \\
\text { regularize } \\
\text { menstrual cycle }\end{array}$} & \multirow{3}{*}{$\begin{array}{l}\text { Whole } \\
\text { plant }\end{array}$} & Methanol & 177.089 \\
\hline & & & & & & & $\begin{array}{l}\text { Ethyl } \\
\text { acetate }\end{array}$ & 609.2692 \\
\hline & & & & & & & Pet ether & 103.479 \\
\hline 25 & $\begin{array}{l}\text { Scoparia dulcis } \\
\text { Linn. }\end{array}$ & Scrophulariaceae & $\begin{array}{l}\text { Misridana } \\
\text { Chinighur } \\
\text { a }\end{array}$ & $\begin{array}{l}\text { Sweet } \\
\text { broom } \\
\text { weed }\end{array}$ & $\begin{array}{l}\text { Burn, infection, } \\
\text { hypertension, } \\
\text { insect bite, gastric } \\
\text { disorder }\end{array}$ & $\begin{array}{l}\text { Whole } \\
\text { weed }\end{array}$ & $\begin{array}{l}95 \% \\
\text { ethanol }\end{array}$ & 577.8608 \\
\hline 26 & $\begin{array}{l}\text { Terminalia } \\
\text { bellerica } \text { roxb }\end{array}$ & Combretaceae & $\begin{array}{l}\text { Bohera, } \\
\text { Bhaira }\end{array}$ & $\begin{array}{l}\text { Belleric } \\
\text { myrobala } \\
\mathrm{n}\end{array}$ & $\begin{array}{lr}\text { Useful in } & \text { in } \\
\text { hemorrhage; dried } \\
\text { fruit in the } \\
\text { treatment } \\
\text { dysentery }\end{array}$ & Bark & Methanol & 47.21 \\
\hline 27 & Luffa cylindrica & Cucurbitaceae & Dundul & $\begin{array}{l}\text { Sponge } \\
\text { gourd }\end{array}$ & $\begin{array}{l}\text { Used in syphilis, } \\
\text { rheumatism }\end{array}$ & Leaf & Methanol & $\begin{array}{l}38730.08 \\
25 \\
\end{array}$ \\
\hline 28 & $\begin{array}{l}\text { Momordica } \\
\text { cochinchinensis }\end{array}$ & Cucurbitaceae & Kakrol & $\begin{array}{l}\text { Sweet } \\
\text { gourd }\end{array}$ & $\begin{array}{l}\text { Used in anemia; } \\
\text { seed membrane aid } \\
\text { in relief of dry eye }\end{array}$ & Leaf & Methanol & 38731.4 \\
\hline 29 & $\begin{array}{l}\text { Centella } \\
\text { asiatica }(\mathrm{L} .)\end{array}$ & Apiaceae & Thankuni & $\begin{array}{l}\text { Indian } \\
\text { penny } \\
\text { worth }\end{array}$ & $\begin{array}{l}\text { Leaf juice in } \\
\text { cataract and eye } \\
\text { trouble; whole } \\
\text { plant is tonic }\end{array}$ & $\begin{array}{l}\text { Whole } \\
\text { plant }\end{array}$ & Methanol & 162.18 \\
\hline 30 & $\begin{array}{l}\text { Coccinea } \\
\text { cordifolia Linn. }\end{array}$ & Cucurbitaceae & Telakucha & Ivy gourd & $\begin{array}{l}\text { Treatment of } \\
\text { diabetes; infusion } \\
\text { used in anorexia, } \\
\text { epilepsy, asthma }\end{array}$ & $\begin{array}{l}\text { Whole } \\
\text { herb }\end{array}$ & Methanol & 251.18 \\
\hline
\end{tabular}


DPPH Scavenging Assay of Eighty Four Bangladeshi Medicinal Plants

\begin{tabular}{|c|c|c|c|c|c|c|c|c|}
\hline 31 & $\begin{array}{l}\text { Ipomoea } \\
\text { aquatica Forsk }\end{array}$ & Convolvulaceae & Kalmi sak & $\begin{array}{l}\text { Water } \\
\text { spinach }\end{array}$ & $\begin{array}{l}\text { Plant is useful in } \\
\text { leprosy and fever }\end{array}$ & $\begin{array}{l}\text { Leaf and } \\
\text { stem }\end{array}$ & Methanol & 295.12 \\
\hline 32 & $\begin{array}{l}\text { Lannea } \\
\text { coromandelica } \\
\text { (houtt)Merr. }\end{array}$ & Anacardiaceae & $\begin{array}{l}\text { Jika, } \\
\text { Jiga, } \\
\text { Kamila }\end{array}$ & Wodier & $\begin{array}{lrr}\text { Bark used } & \text { in } \\
\text { leprous } & \text { and } \\
\text { obstinate } & \text { ulcer; } \\
\text { decoction } & \text { in } \\
\text { toothache } & \\
\end{array}$ & Bark & methanol & 21.37 \\
\hline 33 & $\begin{array}{l}\text { Lawsonia } \\
\text { inermis }(\mathrm{L} .)\end{array}$ & Lythraceae & Mehedi & Samphire & $\begin{array}{l}\text { Leaf paste used in } \\
\text { skin disease }\end{array}$ & Leaf & Methanol & 83.37 \\
\hline 34 & $\begin{array}{l}\text { Nymphaea } \\
\text { nouchalli } \\
\text { Burm.f. }\end{array}$ & Nymphaceae & $\begin{array}{l}\text { Shapla, } \\
\text { Shaluk } \\
\text { gachh }\end{array}$ & Water lily & $\begin{array}{l}\text { Powered form in } \\
\text { piles and } \\
\text { dysentery; show } \\
\text { good activity in } \\
\text { cough }\end{array}$ & Rhizome & Methanol & 75.85 \\
\hline 35 & $\begin{array}{l}\text { Oxalis } \\
\text { corniculata } \\
\text { Linn. }\end{array}$ & Oxalidaceae & Amrul & $\begin{array}{l}\text { Wood } \\
\text { sorrel }\end{array}$ & $\begin{array}{l}\text { Leaf juice in } \\
\text { dysentery; leaf } \\
\text { juice with onion } \\
\text { removes corns and } \\
\text { warts }\end{array}$ & Leaf & Methanol & 162.18 \\
\hline 36 & $\begin{array}{l}\text { Persicaria } \\
\text { hydropiper (L.) } \\
\text { Spach }\end{array}$ & Polygonaceae & Pakarmul & $\begin{array}{l}\text { Smart } \\
\text { weed }\end{array}$ & $\begin{array}{l}\text { Leaf } \\
\text { inflammation, } \\
\text { stomachic, } \\
\text { Seed: carminative, } \\
\text { stimulant }\end{array}$ & $\begin{array}{l}\text { Whole } \\
\text { plant }\end{array}$ & Methanol & 34.8623 \\
\hline 37 & $\begin{array}{l}\text { Phyllanthus } \\
\text { fraternus } \\
\text { auct.Non linn. }\end{array}$ & Euphorbiaceae & $\begin{array}{l}\text { Bhuin } \\
\text { amla }\end{array}$ & $\begin{array}{l}\text { Blackcatn } \\
\text { ip }\end{array}$ & $\begin{array}{l}\text { Treatment } \\
\text { jaundice, } \\
\text { leucorrhea, } \\
\text { stomachache, } \\
\text { dyspepsia }\end{array}$ & $\begin{array}{l}\text { Whole } \\
\text { plant }\end{array}$ & Ethanol & 21.387 \\
\hline \multirow{3}{*}{38} & \multirow{3}{*}{$\begin{array}{l}\text { Triumfetta } \\
\text { rhomboidae } \\
\text { N.Jacq }\end{array}$} & \multirow{3}{*}{ Tillaceae } & \multirow{3}{*}{ Ban okra } & \multirow{3}{*}{$\begin{array}{l}\text { Burbush, } \\
\text { Burweed }\end{array}$} & \multirow{3}{*}{$\begin{array}{l}\text { Fruits and leaves } \\
\text { as demulcent and } \\
\text { astringent }\end{array}$} & Leaf & Ethanol & 65.748 \\
\hline & & & & & & Bark & Ethanol & 22.851 \\
\hline & & & & & & Root & Ethanol & 45.5132 \\
\hline \multirow{4}{*}{39} & \multirow{4}{*}{$\begin{array}{l}\text { Celastrus } \\
\text { paniculatus } \\
\text { willd. }\end{array}$} & \multirow{4}{*}{ Celastraceae } & \multirow{4}{*}{ Shonkhu } & \multirow{4}{*}{$\begin{array}{l}\text { Black oil } \\
\text { tree }\end{array}$} & \multirow{4}{*}{$\begin{array}{l}\text { In the treatment of } \\
\text { epilepsy, gout, } \\
\text { rheumatism; seed } \\
\text { used as anti emetic }\end{array}$} & \multirow{4}{*}{ Seed } & Methanol & 35.9439 \\
\hline & & & & & & & Ethanol & 33.8060 \\
\hline & & & & & & & Pet ether & 35.9439 \\
\hline & & & & & & & Water & 35.9439 \\
\hline 40 & $\begin{array}{l}\text { Casuarina } \\
\text { littorea (L.) }\end{array}$ & Casurinaceae & $\begin{array}{l}\text { Jhau, } \\
\text { Belati } \\
\text { jhau }\end{array}$ & She-oak. & $\begin{array}{l}\text { Bark is astringent } \\
\text { and has traditional } \\
\text { use in diarrhea }\end{array}$ & Bark & Ethanol & 14.467 \\
\hline 41 & $\begin{array}{l}\text { Glinus } \\
\text { oppositifolius } \\
\text { (L.) } \\
\end{array}$ & Molluginaceae & $\begin{array}{l}\text { Jima, } \\
\text { Gima }\end{array}$ & $\begin{array}{l}\text { Sweet } \\
\text { juice }\end{array}$ & $\begin{array}{l}\text { Used to abdominal } \\
\text { pain, jaundice, } \\
\text { inflammation }\end{array}$ & $\begin{array}{l}\text { Whole } \\
\text { plant }\end{array}$ & Methanol & 489.77 \\
\hline 42 & $\begin{array}{l}\text { Cardiospermum } \\
\text { halicacabum } \\
\text { (L.) }\end{array}$ & Sapindaceae & $\begin{array}{l}\text { Kopal } \\
\text { Futki }\end{array}$ & Heart pea & $\begin{array}{l}\text { Traditional } \\
\text { medicine for } \\
\text { rheumatism, } \\
\text { nervous disease, } \\
\text { demulcent,malaria }\end{array}$ & $\begin{array}{l}\text { Whole } \\
\text { plant }\end{array}$ & Methanol & 38.2241 \\
\hline \multirow{3}{*}{43} & \multirow{3}{*}{$\begin{array}{l}\text { Calamus tenuis } \\
\text { Roxb }\end{array}$} & \multirow{3}{*}{ Arecaceae } & \multirow{3}{*}{$\begin{array}{ll}\text { Bet, Jail } \\
\text { bet, } & \text { Pati } \\
\text { bet } & \end{array}$} & \multirow{3}{*}{ Rattan } & \multirow{3}{*}{$\begin{array}{l}\text { Useful in diarrhea; } \\
\text { used as vegetables } \\
\text { for edema, } \\
\text { intrinsic } \\
\text { hemorrhage }\end{array}$} & \multirow{3}{*}{ Fruit } & Methanol & 29.7941 \\
\hline & & & & & & & Pet ether & 32.6258 \\
\hline & & & & & & & $\begin{array}{l}\text { Ethyl } \\
\text { acetate }\end{array}$ & 67.5115 \\
\hline 44 & $\begin{array}{l}\text { Trema } \\
\text { orientalis (L.) }\end{array}$ & Ulmaceae & $\begin{array}{l}\text { Jibon } \\
\text { gachh }\end{array}$ & $\begin{array}{l}\text { Indian } \\
\text { nettle tree }\end{array}$ & 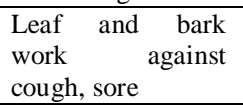 & Leaf & Methanol & 110.25 \\
\hline \multirow[b]{3}{*}{45} & \multirow{3}{*}{$\begin{array}{l}\text { Musa sapientum } \\
\text { L.subsp.sylvestr } \\
\text { is. }\end{array}$} & \multirow[b]{3}{*}{ Musaceae } & \multirow[b]{3}{*}{ Ramkola } & \multirow{3}{*}{$\begin{array}{l}\text { Apple } \\
\text { banana }\end{array}$} & \multirow{3}{*}{$\begin{array}{l}\text { Used in diarrhea, } \\
\text { dysentery, } \\
\text { ulcerative colitis, } \\
\text { diabetes, } \\
\text { hypertension, gout }\end{array}$} & Peel & Methanol & 5907.53 \\
\hline & & & & & & Pulp & Methanol & 95669.52 \\
\hline & & & & & & Seed & Methanol & 54.92 \\
\hline \multirow[b]{2}{*}{46} & \multirow{2}{*}{$\begin{array}{l}\text { Hedyotis } \\
\text { corymbosa (L.) }\end{array}$} & \multirow[b]{2}{*}{ Rubiaceae } & \multirow[b]{2}{*}{ Pitpapra } & Diamond & Applied in fever, & Whole & Methanol & 48.4076 \\
\hline & & & & flower & $\begin{array}{l}\text { depression, } \\
\text { flatulence }\end{array}$ & plant & Pet ether & 44.0618 \\
\hline 47 & $\begin{array}{l}\text { Andrographis } \\
\text { paniculata }\end{array}$ & Acanthaceae & Chirota & $\begin{array}{l}\text { Green } \\
\text { chirota }\end{array}$ & $\begin{array}{l}\text { Exhibit anti fungal, } \\
\text { anti malarial, anti } \\
\text { inflammatory }\end{array}$ & $\begin{array}{l}\text { Whole } \\
\text { Plant }\end{array}$ & Methanol & 414.245 \\
\hline & & & & & Green plant eaten & & Methanol & 58.2666 \\
\hline 48 & $\begin{array}{l}\text { Spinacea } \\
\text { oleracea } \text { Linn. }\end{array}$ & Amaranthaceae & $\begin{array}{l}\text { Palong } \\
\text { shak }\end{array}$ & $\begin{array}{l}\text { Garden } \\
\text { spinach }\end{array}$ & $\begin{array}{l}\text { for urinary calculi } \\
\text { and act as mild } \\
\text { laxative }\end{array}$ & $\begin{array}{l}\text { Whole } \\
\text { plant }\end{array}$ & Water & 149.607 \\
\hline 49 & $\begin{array}{l}\text { Amaranthus } \\
\text { blitum (L.) }\end{array}$ & Amaranthaceae & Lalshak & $\begin{array}{l}\text { Joseph's } \\
\text { coat }\end{array}$ & $\begin{array}{ll}\text { Plant used } & \text { as } \\
\text { astringent } & \text { in }\end{array}$ & $\begin{array}{l}\text { Whole } \\
\text { plant }\end{array}$ & Methanol & 68.8376 \\
\hline
\end{tabular}




\begin{tabular}{|c|c|c|c|c|c|c|c|c|}
\hline & & & & & $\begin{array}{lr}\text { ulcerated mouth } \\
\text { and throat; } \\
\text { mouthwash for } \\
\text { ulcer and sores }\end{array}$ & & Water & 1780.216 \\
\hline \multirow{2}{*}{50} & \multirow{2}{*}{$\begin{array}{l}\text { Daucus carota } \\
\text { (L.) }\end{array}$} & \multirow[t]{2}{*}{ Apiaceae } & \multirow[t]{2}{*}{ Gazor } & \multirow[t]{2}{*}{ Carrot } & \multirow{2}{*}{$\begin{array}{lr}\text { Possess } & \text { good } \\
\text { activity } & \text { against } \\
\text { tumor, piles } & \\
\end{array}$} & \multirow{2}{*}{ Fruit } & Methanol & 69.373 \\
\hline & & & & & & & Water & 146.3943 \\
\hline \multirow[b]{2}{*}{51} & \multirow{2}{*}{$\begin{array}{l}\text { Brassica } \\
\text { olearacea } \\
\text { Var.capitata } \\
\end{array}$} & \multirow[b]{2}{*}{ Cruciferae } & \multirow{2}{*}{$\begin{array}{l}\text { Badha } \\
\text { kopi }\end{array}$} & \multirow[b]{2}{*}{ Cabbage } & \multirow{2}{*}{$\begin{array}{l}\text { Cabbage leaves are } \\
\text { used to treat acute } \\
\text { inflammation }\end{array}$} & \multirow{2}{*}{$\begin{array}{l}\text { Plant } \\
\text { without } \\
\text { root }\end{array}$} & Water & 190.636 \\
\hline & & & & & & & Ethanol & 47.472 \\
\hline \multirow{3}{*}{52} & \multirow{3}{*}{$\begin{array}{l}\text { Polyalthia } \\
\text { suberosa }\end{array}$} & \multirow{3}{*}{ Annonaceae } & \multirow{3}{*}{ Kukuriam } & \multirow{3}{*}{$\begin{array}{l}\text { Corky } \\
\text { debber } \\
\text { tree }\end{array}$} & \multirow{3}{*}{$\begin{array}{l}\text { Act against } \\
\text { microbial } \\
\text { infection, } \\
\text { inflammation in } \\
\text { eye }\end{array}$} & Leaf & $\begin{array}{l}\text { Hydrometh } \\
\text { anol }\end{array}$ & 161.365 \\
\hline & & & & & & Bark & $\begin{array}{l}\text { Hydrometh } \\
\text { anol }\end{array}$ & 65.265 \\
\hline & & & & & & Fruit & $\begin{array}{l}\text { Hydrometh } \\
\text { anol }\end{array}$ & 102.126 \\
\hline 53 & $\begin{array}{l}\text { Trichosanthes } \\
\text { dioica Roxb }\end{array}$ & Cucurbitaceae & $\begin{array}{l}\text { Potol } \\
\text { Palval }\end{array}$ & $\begin{array}{l}\text { Pointed } \\
\text { gourd }\end{array}$ & $\begin{array}{l}\text { Used in } \\
\text { inflammation, } \\
\text { jaundice; act as } \\
\text { antipyretic, } \\
\text { diuretic, laxative } \\
\end{array}$ & $\begin{array}{l}\text { Aerial } \\
\text { Part }\end{array}$ & $\begin{array}{l}\text { Water, } \\
\text { Ethyl } \\
\text { acetate, } \\
\text { Pet ether, } \\
\text { Methanol } \\
\end{array}$ & Very low \\
\hline \multirow[b]{2}{*}{54} & \multirow[b]{2}{*}{ Aloe indica (L.) } & \multirow[b]{2}{*}{ Amaryllidaceae } & \multirow[b]{2}{*}{$\begin{array}{l}\text { Ghrita } \\
\text { kumari }\end{array}$} & \multirow[b]{2}{*}{$\begin{array}{l}\text { Indian } \\
\text { aloe }\end{array}$} & \multirow[b]{2}{*}{$\begin{array}{l}\text { Juice of leaf act in } \\
\text { stomachic; plant is } \\
\text { useful in the } \\
\text { treatment of piles } \\
\text { and fissure, } \\
\text { menstrual } \\
\text { suppression }\end{array}$} & \multirow[b]{2}{*}{$\begin{array}{l}\text { Plant } \\
\text { material } \\
\text { without } \\
\text { leaf }\end{array}$} & Water & 189.753 \\
\hline & & & & & & & Methanol & 99.163 \\
\hline \multirow[b]{3}{*}{55} & \multirow[b]{3}{*}{$\begin{array}{l}\text { Madhuca } \\
\text { longifolia }\end{array}$} & \multirow[b]{3}{*}{ Sapotaceae } & \multirow[b]{3}{*}{$\begin{array}{l}\text { Mahwa, } \\
\text { Mahua }\end{array}$} & \multirow[b]{3}{*}{ Butter tree } & \multirow{3}{*}{$\begin{array}{l}\text { Seed cake is good } \\
\text { fertilizer; } \\
\text { applied in skin, } \\
\text { useful } \\
\text { constipation, } \\
\text { hemorrhoid }\end{array}$} & Fruit & Methanol & 217.3437 \\
\hline & & & & & & & Methanol & 346.1518 \\
\hline & & & & & & Seed & Pet ether & 172.125 \\
\hline 56 & $\begin{array}{l}\text { Vitex negundo } \\
\text { (L) }\end{array}$ & Verbenaceae & $\begin{array}{l}\text { Nishinda, } \\
\text { Samalu }\end{array}$ & $\begin{array}{l}\text { Negundo } \\
\text { chaste tree }\end{array}$ & $\begin{array}{l}\text { Used in headache; } \\
\text { juice of fresh } \\
\text { leaves removes } \\
\text { worms from ulcer }\end{array}$ & Leaf & Ethanol & 81.080 \\
\hline 57 & Momordica & Cucurbitaceae & Corolla & Bitter & $\begin{array}{l}\text { Bitter melon is } \\
\text { used as a folk } \\
\text { medicine in to treat }\end{array}$ & Fruit & Methanol & $\begin{array}{l}1685.6(\text { ho } \\
t \\
\text { extraction } \\
\text { n) }\end{array}$ \\
\hline 51 & charantea & Cucurbitaceae & Corona & gourd & $\begin{array}{l}\text { gastrointestinal } \\
\text { disease and } \\
\text { diabetes }\end{array}$ & Fruit & Mretnanor & $\begin{array}{l}4185.6(\mathrm{co} \\
\text { ld } \\
\text { extraction } \\
\text { ) }\end{array}$ \\
\hline 58 & $\begin{array}{l}\text { Borassus } \\
\text { flabellifer (L.) }\end{array}$ & Arecaceae & Tal gachh & $\begin{array}{l}\text { Palmyra } \\
\text { palm tree }\end{array}$ & $\begin{array}{l}\text { Root has } \\
\text { anthelmintic } \\
\text { property }\end{array}$ & Root & Ethanol & 15.755 \\
\hline 59 & $\begin{array}{l}\text { Stephania } \\
\text { japonica } \\
\text { (Thumb) Miers. }\end{array}$ & Menispermaceae & $\begin{array}{l}\text { Akanadi, } \\
\text { Nimuka }\end{array}$ & $\begin{array}{l}\text { Snake } \\
\text { vine, } \\
\text { Tape vine }\end{array}$ & $\begin{array}{l}\text { Leaves and roots } \\
\text { are bitter and } \\
\text { astringent and used } \\
\text { in fever, diarrhea, } \\
\text { dyspepsia }\end{array}$ & $\begin{array}{l}\text { Whole } \\
\text { plant }\end{array}$ & Ethanol & 422.321 \\
\hline 60 & $\begin{array}{l}\text { Lablab } \\
\text { purpureus } \\
\text { subsp.bengalens } \\
\text { is. }\end{array}$ & fabaceae & $\begin{array}{l}\text { Sadiron } \\
\text { sim }\end{array}$ & $\begin{array}{l}\text { Bean, } \\
\text { Indian } \\
\text { butter } \\
\text { bean }\end{array}$ & $\begin{array}{l}\text { Anthelmintic; used } \\
\text { to treat infection }\end{array}$ & Bin pod & Methanol & 2511.85 \\
\hline 61 & $\begin{array}{l}\text { Smilax } \\
\text { zeylanica Linn. }\end{array}$ & Liliaceae & $\begin{array}{l}\text { Kumarica, } \\
\text { Kumarilat } \\
\text { a }\end{array}$ & $\begin{array}{l}\text { Black } \\
\text { creeper, } \\
\text { Wild } \\
\text { sarsaparill } \\
\text { a }\end{array}$ & $\begin{array}{lr}\text { Roots used in the } \\
\text { treatment } & \text { of } \\
\text { veneral disease; } \\
\text { decoction } & \text { is } \\
\text { applied } & \text { for } \\
\text { rheumatism } & \end{array}$ & $\begin{array}{l}\text { Whole } \\
\text { plant }\end{array}$ & Pet ether & 11274.75 \\
\hline 62 & $\begin{array}{l}\text { Lablab } \\
\text { purpureus }\end{array}$ & Fabaceae & $\begin{array}{l}\text { Beguni } \\
\operatorname{sim}\end{array}$ & $\begin{array}{l}\text { Lablab } \\
\text { bin }\end{array}$ & $\begin{array}{l}\text { Leaf juice and root } \\
\text { are used to treat } \\
\text { infected area }\end{array}$ & Bin pod & Methanol & 1298.263 \\
\hline 63 & $\begin{array}{l}\text { Nyctanthes } \\
\text { arbor-tristis }\end{array}$ & Oleaceae & $\begin{array}{l}\text { Shefali, } \\
\text { Sheuli }\end{array}$ & $\begin{array}{l}\text { Night } \\
\text { flowering } \\
\text { jasmine }\end{array}$ & $\begin{array}{l}\text { Paste of leaves is } \\
\text { used specifically in } \\
\text { treatment of ring } \\
\text { worm }\end{array}$ & $\begin{array}{l}\text { Leaf } \\
\text { ethanol }\end{array}$ & Ethanol & 133.116 \\
\hline 64 & $\begin{array}{l}\text { Psidium } \\
\text { guaiava }(\mathrm{L} .)\end{array}$ & Myrtaceae & $\begin{array}{l}\text { Peyara, } \\
\text { Goam }\end{array}$ & Guava & $\begin{array}{ll}\text { Leaves used } & \text { as } \\
\text { astringent } & \text { for } \\
\end{array}$ & Leaf & Ethanol & 48.906 \\
\hline
\end{tabular}


DPPH Scavenging Assay of Eighty Four Bangladeshi Medicinal Plants

\begin{tabular}{|c|c|c|c|c|c|c|c|c|}
\hline & & & & & bowels, wounds & & & \\
\hline \multirow{3}{*}{65} & \multirow{3}{*}{$\begin{array}{l}\text { Rumex } \\
\text { maritimus }\end{array}$} & \multirow{3}{*}{ Polygonaceae } & \multirow{3}{*}{$\begin{array}{l}\text { Bon } \\
\text { palong }\end{array}$} & \multirow{3}{*}{$\begin{array}{l}\text { Golden } \\
\text { dock }\end{array}$} & \multirow{3}{*}{$\begin{array}{l}\text { Plant is useful in } \\
\text { the treatment of } \\
\text { cough, and } \\
\text { pertussis; paste of } \\
\text { leaf is applied to } \\
\text { swelling }\end{array}$} & \multirow{3}{*}{$\begin{array}{l}\text { Whole } \\
\text { plant }\end{array}$} & $\begin{array}{l}\text { Ethyl } \\
\text { acetate }\end{array}$ & 1369.493 \\
\hline & & & & & & & Pet ether & 1309.318 \\
\hline & & & & & & & Methanol & 416.552 \\
\hline \multirow{3}{*}{66} & \multirow{3}{*}{$\begin{array}{l}\text { Cocculus } \\
\text { hirsutus } \quad \text { (L.) } \\
\text { Diels }\end{array}$} & \multirow{3}{*}{$\begin{array}{l}\text { Menispermiacea } \\
\mathrm{e}\end{array}$} & \multirow{3}{*}{ Jalajmoni } & \multirow{3}{*}{$\begin{array}{l}\text { Broom } \\
\text { creeper, } \\
\text { Ink berry }\end{array}$} & \multirow{3}{*}{$\begin{array}{l}\text { Juice of leaf mixed } \\
\text { with water used as } \\
\text { cooling and } \\
\text { soothing medicine } \\
\text { for gonorrhea }\end{array}$} & \multirow{3}{*}{ Leaf } & $\begin{array}{l}\text { Ethyl } \\
\text { acetate }\end{array}$ & 287.960 \\
\hline & & & & & & & Pet ether & 1701.19 \\
\hline & & & & & & & Methanol & 257.419 \\
\hline 67 & $\begin{array}{l}\text { Eclipta prostata } \\
\text { (L.) }\end{array}$ & Compositae & $\begin{array}{l}\text { Kalokeshi } \\
\text {, Keshraj }\end{array}$ & $\begin{array}{l}\text { False } \\
\text { daisy }\end{array}$ & $\begin{array}{l}\text { Plant is useful in } \\
\text { liver and gall } \\
\text { bladder ailment } \\
\text { and act as } \\
\text { anthelmintic }\end{array}$ & Leaf & Methanol & 27.00 \\
\hline 68 & $\begin{array}{l}\text { Derris trifolia } \\
\text { Lour. }\end{array}$ & Fabaceae & $\begin{array}{l}\text { Gila lata, } \\
\text { Pan lata }\end{array}$ & Derris & $\begin{array}{l}\text { Bark is stimulant, } \\
\text { antispasmodic, and } \\
\text { counter irritant }\end{array}$ & $\begin{array}{l}\text { Whole } \\
\text { plant }\end{array}$ & Methanol & 287.611 \\
\hline \multirow[b]{2}{*}{69} & \multirow[b]{2}{*}{ Thea sinensis } & \multirow[b]{2}{*}{ Theaceae } & \multirow[b]{2}{*}{ Cha } & \multirow[b]{2}{*}{ White tea } & \multirow{2}{*}{$\begin{array}{l}\text { Treating infection } \\
\text { of digestive tract; } \\
\text { leaves are CNS } \\
\text { stimulant }\end{array}$} & \multirow[b]{2}{*}{ Leaf } & Water & 23.333 \\
\hline & & & & & & & Ethanol & 20 \\
\hline 70 & $\begin{array}{l}\text { Phyllanthus } \\
\text { acidus (L.) }\end{array}$ & Euphorbiaceae & $\begin{array}{l}\text { Orbori, } \\
\text { Horbori }\end{array}$ & $\begin{array}{l}\text { Star goose } \\
\text { berry }\end{array}$ & $\begin{array}{l}\text { Fruit is tonic to } \\
\text { liver and used in } \\
\text { bronchitis piles; it } \\
\text { is useful in } \\
\text { vomiting }\end{array}$ & Fruit & Pet ether & 1382.204 \\
\hline 71 & $\begin{array}{l}\text { Capsicum } \\
\text { апnum }\end{array}$ & Solanaceae & $\begin{array}{l}\text { Marich, } \\
\text { Jhal } \\
\text { morich }\end{array}$ & $\begin{array}{l}\text { Chilies, } \\
\text { Red } \\
\text { pepper }\end{array}$ & $\begin{array}{l}\text { Capsicum is used } \\
\text { to treat arthritis } \\
\text { and chilbans to } \\
\text { colic }\end{array}$ & Fruit part & Ethanol & 1849.076 \\
\hline 72 & $\begin{array}{l}\text { Boerhavia } \\
\text { diffusa }\end{array}$ & Nyctaginaceae & Purnalata & Pig-weed & $\begin{array}{l}\text { Root are useful in } \\
\text { jaundice, anemia, } \\
\text { ascites, ophthalmia }\end{array}$ & Root & Methanol & 902.901 \\
\hline 73 & $\begin{array}{l}\text { Acalypha indica } \\
\text { Linn. }\end{array}$ & Euphorbiaceae & $\begin{array}{l}\text { Muktajhur } \\
\text { i }\end{array}$ & $\begin{array}{l}\text { Indian } \\
\text { nettle }\end{array}$ & $\begin{array}{l}\text { Plant is effective in } \\
\text { bronchitis; fresh } \\
\text { leaf juice is useful } \\
\text { in arthritis and } \\
\text { scabies }\end{array}$ & $\begin{array}{l}\text { Whole } \\
\text { plant }\end{array}$ & Ethanol & 484.1279 \\
\hline 74 & $\begin{array}{l}\text { Cassia fistula } \\
\text { Linn. }\end{array}$ & Caesalpiniaceae & $\begin{array}{l}\text { Badar } \\
\text { lathi, } \\
\text { Sonalu }\end{array}$ & $\begin{array}{l}\text { Golden } \\
\text { shower }\end{array}$ & $\begin{array}{l}\text { Pulp of fruit is } \\
\text { laxative and used } \\
\text { in heart disease }\end{array}$ & Bark & Ethanol & 233.1 \\
\hline 75 & $\begin{array}{l}\text { Saraca indica } \\
\text { Linn. }\end{array}$ & Caesalpiniaceae & Ashok & $\begin{array}{l}\text { Asoka } \\
\text { tree }\end{array}$ & $\begin{array}{l}\text { Bark is used in } \\
\text { menorrhagia, cures } \\
\text { biliousness, } \\
\text { dyspepsia }\end{array}$ & Bark & Ethanol & 484.1279 \\
\hline 76 & $\begin{array}{l}\text { Caesalpiniacea } \\
e \quad \text { bonduc } \\
\text { (Linn.) Roxb }\end{array}$ & Caesalpiniaceae & $\begin{array}{l}\text { Nata } \\
\text { karanja }\end{array}$ & $\begin{array}{l}\text { Molucca } \\
\text { bean }\end{array}$ & $\begin{array}{l}\text { Decoction of seeds } \\
\text { useful against } \\
\text { asthma, effective } \\
\text { in blood dysentery }\end{array}$ & Seed & Ethanol & 1561.323 \\
\hline \multirow{6}{*}{77} & \multirow{6}{*}{$\begin{array}{l}\text { Alocasia } \\
\text { decipiens }\end{array}$} & \multirow{6}{*}{ Araceae } & \multirow{6}{*}{$\begin{array}{l}\text { Bish } \\
\text { kachu }\end{array}$} & & \multirow{6}{*}{$\begin{array}{l}\text { This plant claimed } \\
\text { to have analgesic } \\
\text { and and } \\
\text { inflammatory } \\
\text { property }\end{array}$} & \multirow[t]{3}{*}{ Trunk } & $\begin{array}{l}\text { Ethyl } \\
\text { acetate }\end{array}$ & 274.478 \\
\hline & & & & & & & Pet ether & 696.276 \\
\hline & & & & & & & Methanol & 499.518 \\
\hline & & & & & & \multirow{3}{*}{ Root } & $\begin{array}{l}\text { Ethyl } \\
\text { acetate }\end{array}$ & 640.395 \\
\hline & & & & & & & Pet ether & 665.888 \\
\hline & & & & & & & Methanol & 171.862 \\
\hline 78 & $\begin{array}{l}\text { Bacopa } \\
\text { monniera } \\
\text { pennel }\end{array}$ & Scrophulariaceae & $\begin{array}{l}\text { Brhami } \\
\text { sak }\end{array}$ & $\begin{array}{l}\text { Herb of } \\
\text { grace }\end{array}$ & $\begin{array}{l}\text { Plant is used for } \\
\text { neuralgia, } \\
\text { epilepsy, and } \\
\text { mental illness }\end{array}$ & $\begin{array}{l}\text { Whole } \\
\text { plant }\end{array}$ & Ethanol & 401.0093 \\
\hline 79 & $\begin{array}{l}\text { Albizia lebbeck } \\
\text { (Linn.) Benth. }\end{array}$ & Mimosaceae & $\begin{array}{l}\text { Shirish, } \\
\text { Koroi }\end{array}$ & $\begin{array}{l}\text { Flea tree, } \\
\text { Parrot tree }\end{array}$ & $\begin{array}{lr}\text { Bark cures } \\
\text { toothache and } \\
\text { disease of gum }\end{array}$ & Bark & Ethanol & 401.0093 \\
\hline \multirow[b]{3}{*}{80} & \multirow{3}{*}{$\begin{array}{l}\text { Coriandrum } \\
\text { sativum }(L .)\end{array}$} & & & & Infusion of the & & Water & 591.812 \\
\hline & & & Dhonia, & & herb is gentle & & Pet. ether & 758.046 \\
\hline & & umbelliferae & Dhoney & Coriander & $\begin{array}{ll}\text { remedy } & \text { for } \\
\text { flatulence, } & \\
\text { bloating, } & \text { and } \\
\text { cramp } & \end{array}$ & Leaf & $\begin{array}{l}\text { Rectified } \\
\text { spirit }\end{array}$ & 450.543 \\
\hline
\end{tabular}


DPPH Scavenging Assay of Eighty Four Bangladeshi Medicinal Plants

\begin{tabular}{|c|c|c|c|c|c|c|c|c|}
\hline 81 & $\begin{array}{l}\text { Enhydra } \\
\text { fluctuans Lour. }\end{array}$ & Compositae & $\begin{array}{l}\text { Hincha } \\
\text { shak }\end{array}$ & $\begin{array}{l}\text { Water } \\
\text { cress }\end{array}$ & $\begin{array}{l}\text { Plant is nutritious } \\
\text { and used in ascites, } \\
\text { dropsy }\end{array}$ & Leaf & Ethanol & 15.653 \\
\hline 82 & $\begin{array}{l}\text { Cassia sophera } \\
\text { Linn. }\end{array}$ & Caesalpiniaceae & $\begin{array}{l}\text { Chhota, } \\
\text { Jhigni }\end{array}$ & $\begin{array}{l}\text { Senna } \\
\text { sohpera }\end{array}$ & $\begin{array}{l}\text { Leaves are used in } \\
\text { cough, asthma, } \\
\text { bronchitis }\end{array}$ & Leaf & Ethanol & 1504.583 \\
\hline 83 & $\begin{array}{l}\text { Minusops elengi } \\
(L .)\end{array}$ & Sapotaceae & Bakul & $\begin{array}{l}\text { Indian } \\
\text { medaller }\end{array}$ & $\begin{array}{l}\text { Bark and fruit are } \\
\text { used in the } \\
\text { treatment } \\
\text { diarrhea }\end{array}$ & Leaf & Methanol & 20.380 \\
\hline 84 & $\begin{array}{l}\text { Opuntia } \\
\text { (Ker. } \\
\text { Haw. }\end{array}$ & Cactaceae & $\begin{array}{l}\text { Phonimon } \\
\text { osa }\end{array}$ & $\begin{array}{l}\text { Pricly } \\
\text { pear }\end{array}$ & $\begin{array}{l}\text { Leaves are } \\
\text { poultice to reduce } \\
\text { inflammation, and } \\
\text { ophthalmia }\end{array}$ & Leaf & $\begin{array}{l}\text { Methanol } \\
(80 \%)\end{array}$ & 22.426 \\
\hline
\end{tabular}

TABLE 2: IC $_{50}$ value of two standards used

\begin{tabular}{|l|l|}
\hline Standard & IC50 value $(\mu \mathrm{g} / \mathrm{mL})$ \\
\hline Ascorbic acid & 15.5 \\
\hline BHT (Butylated Hydroxy Toluene) & 46.54 \\
\hline
\end{tabular}

\section{Discussion}

In traditional society health and nutrition are interconnected and many medicinal plants are consumed both for food and medicinal purposes. This type of consumption played a great role in diet but there are very few ethnopharmacological phytopharmacological studies that dealt with potential health benefits of such diets.

In this study total 84 medicinal plants were evaluated. Free radicals exist in our body with one or more unpaired electrons. Propagation of free radicals can cause thousands of reactions in our body which could result in cell membrane disintegration, protein, and lipid damage, DNA mutation which can lead to propagation of many diseases like cancer, ulcer, diabetes, cardiovascular disorder etc.[8]. Antioxidants play beneficial role by scavenging free radicals.

For medicinal plants, to find out antioxidant potency, DPPH scavenging assay is the procedure[9, 10]. It is a direct test for free radical scavenging. DPPH (purple colored) is a stable free radical which is reduced to $\alpha, \alpha$-diphenyl- $\beta$-picryl hydrazine (yellow colored) by converting unpaired election to paired one. Antioxidants act by converting unpaired election to paired one. DPPH containing odd electron is responsible for the absorbance at $517 \mathrm{~nm}$ and also for a deep purple color and when it gets neutralized by accepting electron from an antioxidant compound it becomes decolorized which can be measured from the change in absorbance.

From this study we could conclude that, around 84 medicinal plants Syzygim cumini, Commelina benghalensis, Lannea coromandelica, Phyllanthus fraternus, Triumfetta rhomboidae, Casuarina littorea, Calamus tenuis, Borassus flabellifer, Artocarpus lacucha, Eclipta prostata, Enhydra fluctuans, Minusops eleng, Opuntia dilleni, and Thea sinensis showed excellent DPPH scavenging property (90\% or more). Syzygim cumini, Casuarina littorea, Borassus flabellifer, Enhydra fluctuans, and Minusops elengi exhibited highest radical scavenging potentiality with an IC50 value of $12.816 \mu \mathrm{g} / \mathrm{mL}, 14.467 \mu \mathrm{g} / \mathrm{mL}, 15.755 \mu \mathrm{g} / \mathrm{mL}$, $15.653 \mu \mathrm{g} / \mathrm{mL}$, and $20.380 \mu \mathrm{g} / \mathrm{mL}$ respectively. These results are presented in the diagram below;

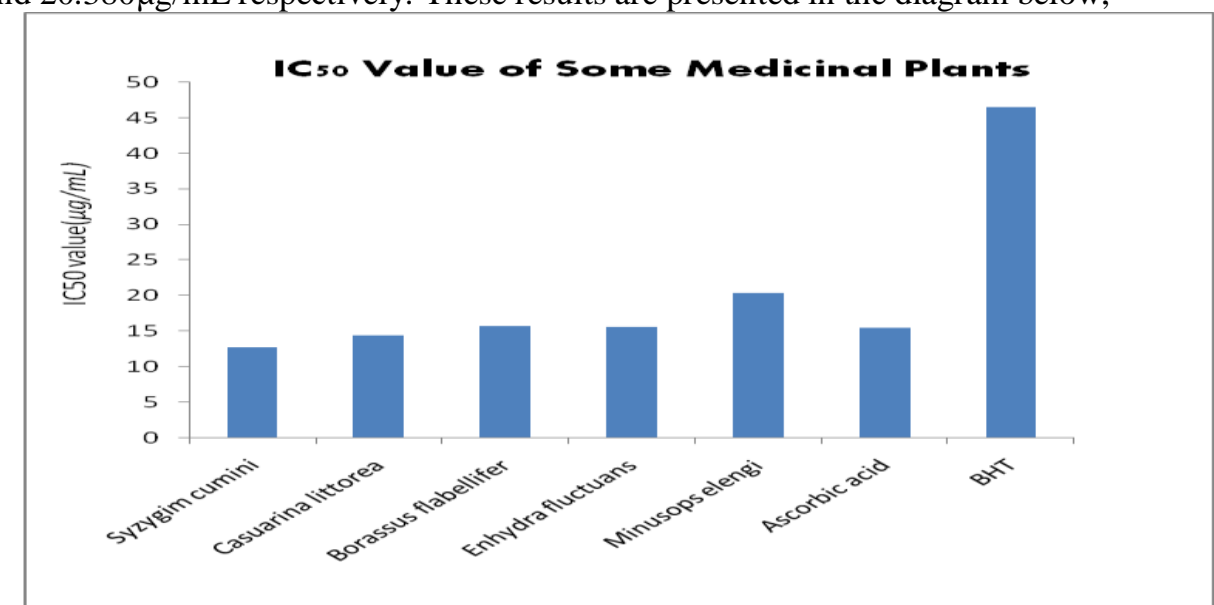

Fig.1: IC50 value of some medicinal plants showing highest scavenging property

All these value are very close to the IC50 value of 15.5 presented by ascorbic acid and better than IC50 value of 46.537 presented by BHT (Butylated Hydroxy Toluene). Syzygim cumini is the most powerful and 
excellent scavenger of DPPH free radical among all tested plants. 30 plants presented good scavenging property. Among them Punica granatum $(35.558 \mu \mathrm{g} / \mathrm{mL}$ ), Commelina benghalensis (aerial part-pet ether fraction $=43.7936$ $\mu \mathrm{g} / \mathrm{mL})$, Cocos nucifera $(41.2227 \mu \mathrm{g} / \mathrm{mL})$, Artocarpus lacucha (pericarp-methanol fraction $=33.501 \mu \mathrm{g} / \mathrm{mL}$ ), Cyperus rotundus $(35.9439 \mu \mathrm{g} / \mathrm{mL})$, Dillenia indica $(32.622 \mu \mathrm{g} / \mathrm{mL})$, Persicaria hydropiper $(34.8623 \mu \mathrm{g} / \mathrm{mL})$, Triumfetta rhomboidae (root-ethanol $=45.5132 \mu \mathrm{g} / \mathrm{mL}$ ), Celastrus paniculatus (seed-methanol $=35.9439 \mu \mathrm{g} / \mathrm{mL}$, seed- Ethanol $=33.8060 \mu \mathrm{g} / \mathrm{mL}$, seed- Pet ether $=35.9439 \mu \mathrm{g} / \mathrm{mL}$, seed- Water $=35.9439 \mu \mathrm{g} / \mathrm{mL}$ ), Cardiospermum halicacabum $(38.2241 \mu \mathrm{g} / \mathrm{mL})$, Calamus tenuis (fruit-pet ether $=32.6258 \mu \mathrm{g} / \mathrm{mL}$ ), and Hedyotis corymbosa (whole part-pet ether $=44.0618 \mu \mathrm{g} / \mathrm{mL}$ ) exhibited good scavenging property than standard reference BHT which presented IC50 value of $46.537 \mu \mathrm{g} / \mathrm{mL}$. 14 plants showed moderate scavenging property between 80 to $150 \mu \mathrm{g} / \mathrm{mL}$, the rest showed low scavenging property. 3 out of 84 medicinal plants presented lowest and very poor scavenging potency. Luffa cylindrical and Momordica cochinchinensis have IC50 value of $38730.0825 \mu \mathrm{g} / \mathrm{mL}$ and $38731.4 \mu \mathrm{g} / \mathrm{mL}$ respectively. Pulp (methanol) fraction of Musa sapientum showed the lowest potency among the 84 medicinal plants with an IC 50 value of $95669.52 \mu \mathrm{g} / \mathrm{mL}$.

It has been stated that radical scavenging property is due to phenolic compounds like flavonoid, polyphenol, and tannin[11]. Free radical neutralizing, quenching singlet or triplet oxygen is due to electron donating activity of phenolic compounds[12]. Oxidative damage is basic reason which leads to various disorders like inflammation, viral inflammation, autoimmune diseases and ulcer[13]. Reactive oxygen species are responsible for triggering carcinogenesis process starting with DNA damage and triggers irregular genetic events which lead to uncontrolled cell division. Finally carcinoma develops[13]. Hence free radical scavengers have potentiality to prevent, delay or stop these disorders. Evaluating the laboratory and epidemiological study of antioxidant potentiality of various plants in last 24 years, scientists experienced that whole edible plants or their active components like flavonoid, tannin, volatile oil, glycoside, alkaloids etc have substantial and protective effect on human carcinogenesis[13, 14, 15].

\section{Conclusion}

From the above result and discussion tested plants have moderate to excellent free radical scavenging activity and selected medicinal plants which have very good antioxidant property can be used as a source of newer antioxidant preparation in future. So, further research project should be run to find out the active components present in these plants which are responsible for playing life saving role in human body.

\section{Acknowledgement}

I am greatly thankful to Dr. Md. Sohel Rana, Professor, Department of Pharmacy, Jahangirnagar University, for providing necessary facilities, logistic support, and continuous encouragement to carry out this research.

\section{References}

[1] Packer L, The antioxidant miracle: Your complete plan for total health and healing, John Wiley \& Sons Inc., Canada, 1999.

[2] Yildirim A, Mavi A, Oktay M, Kara AA, Algur OF, Bilaloglu V, Comparison of antioxidant and antimicrobial activitiesof tilia (Tilia argenta Desf Ex DC), sage (Salvia triloba L.) and black tea (Camellia sinensis) extracts. Journal of Agricultural and Food Chemistry 48 (2000) 5030-5034.

[3] Mantle D, Eddeb F, Pickering A, Comparison of relative antioxidant activities of British medicinal plant species in vitro. Journal of Ethnopharmacology 72 (2000) 47-51.

[4] Campbell IC, Abdulla EM, Strategic approaches to in vitro neurotoxicity, in approaches and methods, in, Neurotoxicology, Academic press London, 1995, pp. 495.

[5] G Cao, ER Sofic, RL Prior, Antioxidant capacity of tea and common vegetables. Journal of Agricultural and Food Chemistry 44 (1996) 3426-3431.

[6] A Ghani, Medicinal plants of Bangladesh 2nd ed., Asiaticsociety of Bangladesh, Dhaka, 2003.

[7] Braca A, Tommasi ND, Bari LD, Pizza C, Politi M, Morelli I, Antioxidant principles from Bauhinia terapotensis. Journal of Natural Products 64 (2001) 892-895.

[8] Liao KL, Yin MC, Individual and combined antioxidant effects of seven phenolic agents in human erythrocyte membrane ghosts and phosphatidylcholine liposome systems: Importance of partition co-efficient. Journal of Agricultural and Food Chemistry 48 (2000) 2266-2270.

[9] Koleva II, Van Beek TA, Linseen JPH, Groot A, Screening of plant extracts for antioxidant activity: A comparative study of three testing methods. Phytochemical Analalysis 13 (2002) 8-17.

[10] Suresh PK, Sucheta S, Sudarshana VD, Selvamani P, Latha S, Antioxidant activity in some selected Indian medicinal plants. African Journal of Biotechnology 7 (2008) 1826-1828.

[11] Rahman MAA, Moon SS, Antioxidant polyphenol glycosides from the plant Draba niomrasa. Bulletin of the Korean Chemical Society 28 (2007) 827-831.

[12] Hasan SM, Hossain, A Faruque, Comparison of antioxidant potential of different fractions of Commelina benghalensis Linn. Bangladesh Journal of Life Science 20 (2008) 9-16.

[13] P Greenwald, Science, medicine, and the future of cancer chemoprevention. British Medical Journal 324 (2002) 714-718.

[14] Tsao TS, Kim ES, Hong WK, Chemoprevention of cancer. CA: A Cancer Journal for Clinicians 54 (2004) 150-180.

[15] Metha RG, Pezzuto JM, Discovery of cancer preventive agents from natural products: from plants to prevention. Current Oncology Reports 4 (2002) 478-486. 